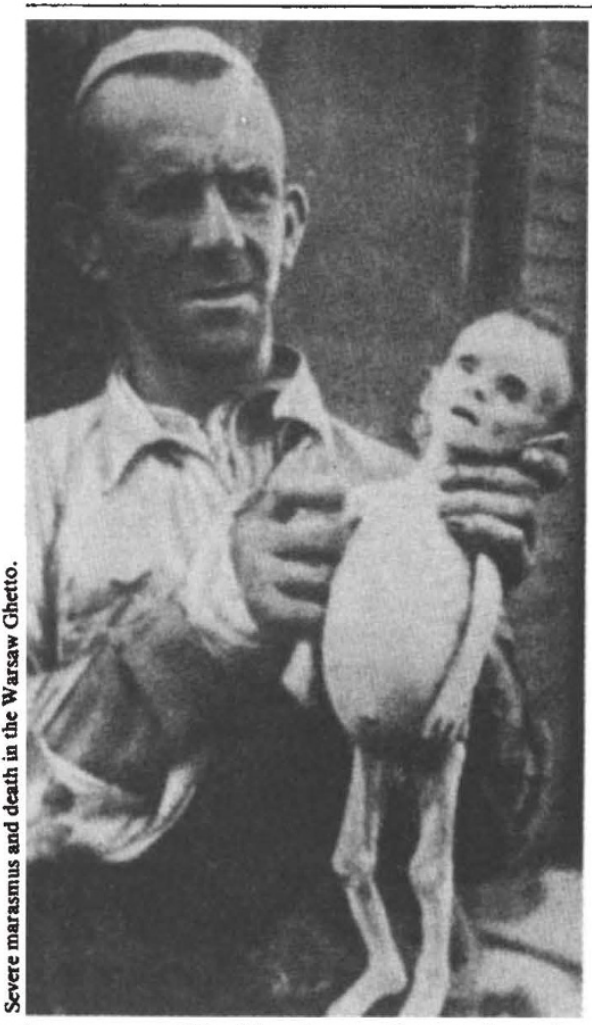

because, unlike Keys' experimental study, it deals with people starving to death.

I am sure that many of the results the authors obtained will still be of scientific interest.
For example, the chapter by Fliederbaum et al. on metabolic changes records that in severely malnourished patients BMR was $30-40 \%$ below normal and was not stimulated by protein feeding, but increased by $20-50 \%$ when sugar was fed. Or again, Fajgenblat's brief report on ocular changes in starvation, or Apfelbaum-Kiwalski's report on the pathophysiology of the circulatory system in starvation, will be read with interest by all workers in the field. All will, like me, be saddened by the brevity of the reports: in reading the book I often found myself wishing that all the authors had reported their raw data, or referenced the exact methods by which they worked out complex indices (like the degree of normality of BMR) but, given the conditions in which they worked, one can only be grateful that anything at all has survived.

That which has survived will be accorded a place of honour amongst many analogous nutritional studies on the pathophysiology of protein-energy malnutrition in children that have been produced since the Second World War.

However there will remain one crucial difference between this book and many other postwar studies on the biochemistry of malnutrition. Most scientists studying malnutrition since the war have done so because they believed that the causes and cures of malnutrition should be sought at the physiological level. The authors of this book had no such illusion. The cause of the malnutrition they describe is to them clear: it is the result of a systematic policy which, in isolating the Jews from the economic life of the nation, sentenced them to death. It was a policy which allowed a Jew only 800 kcal per day, under half of that allowed by the Germans even for people who did no work worth mentioning. It was in response to this policy that the authors undertook their study, not because they believed they would find a scientific cure for the Hunger Disease that this policy induced but because all they could do as scientists was to create a memorial to the dead, by their contribution to scientific knowledge.

Is there in this a lesson for our times? In the 40 years since the Ghetto was destroyed, immense scientific effort has been put into studies of the nutritional and metabolic aspects of protein-energy malnutrition in children without doing anything to reduce the prevalence of the disease. Perhaps the heroic efforts of Milejkowski and his colleagues should cause us now to focus more clearly on the cause of the disease and step outside a narrow scientific paradigm to seek a cure.

John Rivers is a Lecturer in the Department of Human Nutrition at the London School of Hygiene and Tropical Medicine, University of London, UK.

\section{O polish'd perturbation}

\section{A.J. Meadows}

Planets $X$ and Pluto. By William G. Hoyt. Pp.302. (University of Arizona Press: Tucson, Arizona, 1980.) Hardback \$17.50; paperback \$9.50.

A YEAR from now we will be celebrating the two-hundredth anniversary of the discovery of the planet Uranus by William Herschel. Compared with, say, the Einstein centenary which occurred recently this might seem to be an event of limited interest. In terms of its contemporary impact, however, Herschel's discovery attracted as much public attention as the General Theory of Relativity did in this century. For the first time since prehistory, the scope of the Solar System had been enlarged. The natural reaction of astronomers was to look for other, as yet undiscovered, planets - a hunt which lasted for a century and a half.

Mr Hoyt's book is primarily concerned with the investigations that led to the last of these planetary discoveries - the detection of Pluto. But it is logical that he should introduce this search with a detailed discussion of the events surrounding the discovery of earlier planets before turning to Pluto itself. His account is readable, but highly detailed. Like his earlier book,
Lowell and Mars, the narrative is partly based on material in the Lowell archives. Like the earlier book, too, it is intended as a partial history of the Lowell Observatory, and so covers matters other than planets. But it is the search for Planet X - as the supposed planet beyond Neptune was labelled - that dominates the story.

The main outlines of the hunt for new planets is fairly familiar, at least up to the discovery of Neptune. But this earlier history - and especially the problems facing the theoretical prediction of Neptune's position in the sky - provides a fascinating parallel with the subsequent search for Planet X. Neptune was sought because the newly discovered Uranus stubbornly refused to follow its predicted path. The postulate of an outer planet, gravitationally perturbing Uranus, became an increasingly attractive possibility. Ultimately, two theoretical astronomers, Leverrier and Adams, independently predicted a position for this supposed planet. Their results were in good agreement with each other, and Neptune was, indeed, picked up close to the predicted point. So far, this was a major success story. But it rapidly became evident that some of the assumptions made by both Leverrier and Adams in determining the new planet's position were far removed from reality. How, then, had they managed to pinpoint its place so accurately? It was very quickly suggested that their result was purely a 'happy accident', a conclusion which was equally quickly denied. The controversy continues today (it is, perhaps, a slight defect in this book that the subsequent analyses of the problem are not fully covered); but a modern consensus would give Leverrier and Adams the benefit of the doubt.

The search for Planet $\mathbf{X}$ possessed one immediate difference from the hunt for Neptune. Like the latter it was based on an examination of residuals, otherwise unaccounted for, in the orbits of the inner planets (in this case, Uranus and Neptune). The residuals now, however, were much smaller than those that had originally attracted attention to the existence of Neptune. It was less that these residuals forced a search for a new planet than that the desire to find a new planet motivated the investigation of the residuals.

The most detailed, though not the only, attack on the problem of Planet $\mathrm{X}$ was by Percival Lowell, and much of the book revolves round his activities. Lowell's final predictions appeared in 1915 - only a year before his death - but the astronomers at Lowell Observatory had already started on a photographic search for the supposed planet in 1905. After Lowell's death the search lapsed, only to be resumed at the end of the 1920s. It was then undertaken by Tombaugh, a new recruit to the observatory staff, and, early in 1930, he discovered Pluto close to the point indicated by Lowell's calculations.

This sounds like a repeat of the 
successful hunt for Neptune. The similarity extends even further, for one of Lowell's contemporaries, W. H. Pickering, had predicted the existence of a planet at about the same place as that suggested by Lowell. (The parallel with Leverrier and Adams cannot be pushed too far, however, for Pickering enthusiastically predicted the existence of several planets at various distances from the Sun.) There was one major difficulty: both Lowell and Pickering supposed in their calculations that Planet $\mathrm{X}$ must be a reasonably massive body. Pluto, when found, proved to be fainter, and so presumably smaller, than expected. Was it really the predicted planet? The arguments started once again: rather unproductively this time, because no mass was available for Pluto. The climax both of this dispute and of the book has actually only just occurred: for, in 1978, a Plutonian satellite was finally discovered. The consequences are remarkable - to quote Mr. Hoyt:

"The discovery of a Plutonian satellite [named Charon], of course, permitted the first direct and reasonably accurate determination of Pluto's mass since the planet's discovery forty-eight years ago . . . . Pluto is thus a very, very small planet indeed, only about 20 percent as massive as the earth's moon, and with a probable diameter of only 1500 miles $(2420 \mathrm{~km})$, about two-thirds that of the earth's moon.
"The discovery of Charon, and the consequent determination of Pluto's very small mass, made it certain that Pluto could not be Percival Lowell's predicted Planet $\mathrm{X}$, and thus the long controversy over this question ended."

The result is stated firmly; but it leads to the very odd conclusion that the positions of Pluto (certainly) and Nepture (possibly) were correctly predicted by accident. The Einstein centenary reminded us that God does not play dice. Perhaps the Uranus bicentenary should assure us that $\mathrm{He}$ does.

A.J. Meadows is Professor of Astronomy and History of Science at the University of Leicester, UK.

\section{New astronomies}

\section{R.J. Tayler}

A Source Book in Astronomy and Astrophysics, 1900-1975. Edited by K.R. Lang and O. Gingerich. Pp.922. (Harvard University Press: Cambridge, Massachusetts, and London, UK, 1980.)\$50.

THIS book provides an attractive invitation to review the great increase in our knowledge of the Universe over the present century. In 1900, most astronomers believed that that the Milky Way system was the whole Universe and that the Sun was near its centre. The existence of interstellar matter was suspected but not established and it was not known that interstellar dust absorbed starlight and produced a seriously biased view of the Universe. Although spectroscopy had shown that stars contain the same chemical elements as the Earth, a quantitative discussion was impossible because the structure of atoms and the origin of spectral lines were not understood. A study of stellar structure had started but its further development required more knowledge of atomic physics and of nuclear structure, leading to an explanation of the source of stellar energy.

For the first two-thirds of the period under review, observational advances were restricted to optical astronomy. The Universe was seen to be an immense expanding system of galaxies and the structure of our Galaxy and its constituent stars and gas clouds was studied in great detail. Towards the end of this great period of optical astronomy, many astronomers believed that all the important components of the Universe had been discovered. This view has been transformed in the past twenty-five years by the development of new astronomies - radio, infrared, ultraviolet, X-ray and $\gamma$-ray - studying the 'invisible Universe', and by the discovery, for example, of quasars, pulsars, X-ray binaries, interstellar neutral hydrogen and molecules and the cosmic microwave radiation. Theoreticians have used developments in physics, such as quantum theory and general relativity, to explain the properties of objects already known and to predict the existence of new types of object.

Drs Lang and Gingerich have selected about 150 key papers to trace the increase in our knowledge. In some cases the papers are printed in full and in others the less essential parts of the discussion have been paraphrased. In every instance, they have introduced their selection by some general remarks on the development of the subject; this enables them to give due credit for key work which they could not include in the anthology. I find this a fascinating collection of papers and I am impressed by the quality of the editorial work. In reading the book, I have learnt many things which I had not previously known and others which I had forgotten. The book should certainly be widely available to both young and old students of astronomy. It is inevitable that not all astronomers will agree with the choice of key papers. In general, I did find what I expected but I was surprised that, although the work of Chandrasekhar is widely quoted, not one of his papers is included and I would have included Parker's paper on the solar wind.

R.J. Tayler is Professor of Astronomy and Director of the Astronomy Centre at the University of Sussex, Brighton, UK.

\section{CONTINUING CHANGE IN SCIENTIFIC THOUGHT}

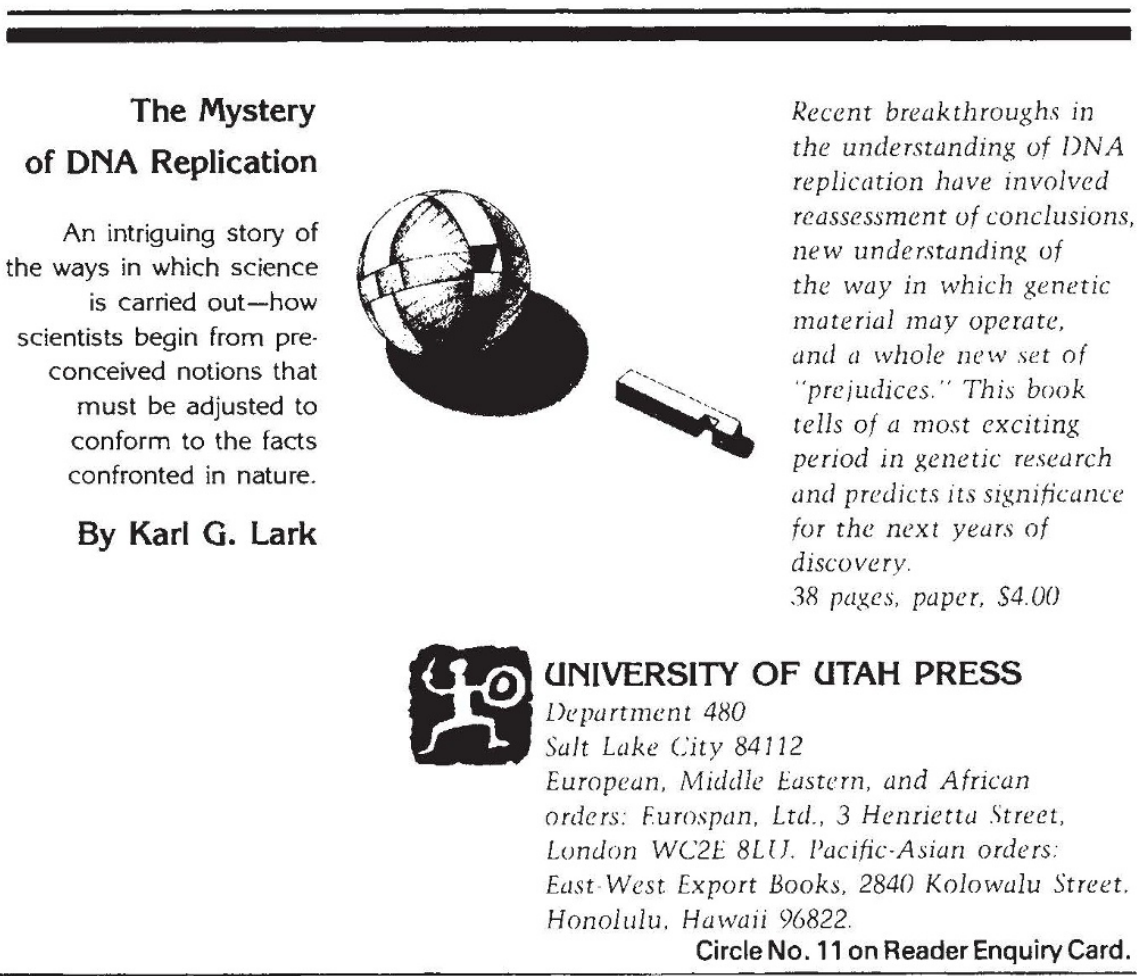

